\title{
Foreword: Predicting the responses of animals to their nutrients - quo vadimus?
}

For its 2006 Annual Meeting BSAS organised an invited session on 'Responses to Nutrients'. The session was appropriately chaired by Professor Colin Whittemore, who was responsible for the first systematic approach to the integration of information about an animal its feed and the environment in which it was kept, with a view to predicting its responses and simulating its performance (Whittemore and Fawcett, 1976). In his usual indomitable fashion Professor Whittemore questioned whether there was a need for such a session. Was there anything that we did not already about know animals responded to their intake of nutrients? After all models of growth for pigs and poultry have in the past 30 years been able not only to predict such responses, but at the same time to deal with the more challenging task of voluntary feed intake prediction. Such models have been used increasingly to optimise feed and feeding programmes in pig and poultry enterprises worldwide. On the other hand, in ruminants many applied feeding models are still largely based on meeting animal requirements.

The remit of the papers presented at the meeting and developed in full in this issue was precisely this: to review the gaps in our knowledge, which inhibit us from making robust predictions on what the outcome of a certain feeding regime would be. An additional aim of the papers was to identify dimensions that need to be taken into account in order to make the predictions of responses to nutrients more applicable or capable of extension. For example, such models are increasingly required to make predictions about the quantity and quality of a saleable product, such as carcass or portion yield, as opposed to the simple predictions of tissue retention (Gous and Berhe, 2006). In addition there is an increasing need to predict product quality for its consequences on human health. On the other hand nutrients are increasingly seen as promoters of animal health and by extension of productivity in challenging environments. In turn disease may influence the manner in which nutrients are partitioned in the body, between disease and production associated functions (Houdijk et al., 2001). These are some of the challenges imposed upon the new generation of models or responses to nutrients!

The first paper in the series by Gous considers the future challenges faced by frameworks developed to predict nutrient responses in poultry. Such challenges include their extension to the prediction of mortality and uniformity in a broiler flock; these dimensions were not considered in the past to be under nutritional control. Other challenges include the correct description of the bird genotypes being simulated, and whether the developed frameworks are capable of predicting the interactions between these genotypes and the environment they are kept in. The conclusion drawn is that for such interactions to be predicted accurately, a better description of the environmental dimensions is called for. Such dimensions include the constraints imposed by the physical and social environment, and infection.

The issue of nutrient partitioning in animals with a variable degree of experience and exposure to pathogens is developed by Sandberg et al.. The paper extends existing frameworks that were able to predict responses to nutrients under different physical and social environmental conditions (Wellock et al., 2003a and b). As suggested by Kyriazakis (1999) in order to predict accurately the behaviour of a system, a sufficient description is required. Thus the consideration of the infectious dimension(s) of the environment can only improve the accuracy of our predictions, as farm animals are raised in environments that include a degree of pathogen challenge and where subclinical infection prevails. The paper identifies two key problems, other than the 'infectivity' of the environment, that would need to be solved to achieve this: (i) an adequate description of the ability of the animal to deal with pathogens; and (ii) the rule or rules animals use to partition nutrients between health and production related functions. By doing so, the paper leads to the designs of relevant experiments that will improve our understanding of the relationship between animal performance and pathogen exposure in different environments.

The paper by Friggens and Newbold considers the prediction of nutrient partitioning during lactation, using the dairy cow as an example. The complexities of the problem are obvious, as one needs to consider the sources and fate of nutrients that derive not only from the food, but also from the animal's body. The authors assert that the interplay between these two sources of nutrients is genetically driven, and thus animal responses cannot be predicted on the basis of food properties alone. The paper thus calls for a better description of the reproducing animal, in terms of what it is trying to achieve both in the short and long term. For such an animal the consequences of nutrient partitioning in one reproductive cycle will not only influence current outcomes, but will result in long term 
consequences on its lifetime productivity. For this reason the authors put forward the idea of trade-offs in the short and long-term nutrient partitioning. The hope is that this approach will allow for prediction of the effects of management and genetic selection for multiple traits on the nutrient partitioning of dairy cows.

The final paper by Dijkstra et al. shares a lot of similarities with the preceding paper, in that it aims to predict the profile of absorbed nutrients from the food in dairy cattle. By doing so it makes the problem of digestion and absorption central to their framework. The difference with the approach of Friggens and Newbold is obvious. The attraction, however, of the approach taken by Dijkstra et al. lies in the fact that their framework can be and is extended to predict the excretion of nutrients in the environment. Their model can thus be extended to include mitigation strategies to reduce excretion of waste into the environment. In the past models of responses to nutrients have considered mainly the fate of food resources of economic importance (such as nitrogen). As environmental concerns are becoming an integral part of the animal supply chain, the partitioning and excretion of macro and microminerals becomes an issue worth accounting for. Thus, the increased importance of environmental issues on dairy cattle production will likely open up new opportunities to develop and improve nutritional models, be it from a different perspective.

On the basis of the above four papers I conclude that in spite of the significant progress made in the past three decades, there are still challenges that lie ahead for those willing to try to predict responses to nutrients in farm animals. As expected, many of the challenges have been raised through the development of existing frameworks, and our desire to improve the accuracy and applicability of the resultant models. As a result, all four papers call for a better or adequate description of the animal and the environment being simulated, to achieve this. Models that are capable of such predictions have the potential to be of immense benefit to animal scientists and by extension the industries they serve.

I. Kyriazakis

Animal Nutrition and Health Department Scottish Agricultural College West Mains Road, Edinburgh EH9 3JG, UK and

Faculty of Veterinary Medicine University of Thessaly 43100 Karditsa, Greece E-mail: Ilias.Kyriazakis@sac.ac.uk

\section{References}

Gous RM and Berhe ET 2006. Modelling populations for purposes of optimisation. In Mechanistic modelling in pig and poultry production (eds RM Gous, TR Morris and C Fisher), pp. 78-96. CABI Publishing, Wallingford, UK.

Houdijk JGM, Jessop NS and Kyriazakis I 2001. Nutrient partitioning between reproductive and immune functions in animals. Proceedings of the Nutrition Society 60, 515-525.

Kyriazakis I 1999. Future directions for models in pig biology. In A quantitative biology of the pig (ed. I Kyriazakis), pp. 381-388, CABI Publishing, Wallingford, UK.

Wellock IJ, Emmans GC and Kyriazakis I 2003a. Modelling the effects of thermal environment and dietary composition on pig performance: model and logic concepts. Animal Science 44, 255-265.

Wellock IJ, Emmans GC and Kyriazakis I 2003b. Predicting the consequences of social stresses on pig feed intake and performance. Journal of Animal Science 81, 2995-3007.

Whittemore CT and Fawcett R 1976. Theoretical aspects of a flexible model to simulate protein and lipid growth in pigs. Animal Production 22, 87-96. 